\title{
Offline Secondary Electron Counting and Conditional Re-illumination in SEM
}

Akshay Agarwal $^{1}$, John Simonaitis ${ }^{1}$, Vivek Goyal ${ }^{2}$ and Karl Berggren ${ }^{1}$

${ }^{1}$ Massachusetts Institute of Technology, Cambridge, Massachusetts, United States, ${ }^{2}$ Boston University, Boston, Massachusetts, United States

Adaptive illumination schemes have recently been implemented in scanning transmission electron microscopy (STEM) and scanning electron microscopy (SEM) to mitigate sample damage during imaging [1,2]. In such schemes, the sample is imaged by illuminating only a fraction of the sample pixels. In STEM, a major enabling factor for these schemes has been the implementation of electron count imaging [3]. We had recently proposed an adaptive scheme called conditional re-illumination (CR) that can significantly reduce sample damage by using scattered electron counting on a STEM or secondary electron (SE) counting in SEM [4]. However, SE counting is not commonly available on an SEM which limits the implementation of this scheme.

In this work, we have implemented SE count imaging and CR offline on an SEM (Zeiss Leo 1525). To implement SE counting, we collected the SE detector signal on a $2 \mathrm{GHz}$ oscilloscope and verified through a study of its statistics that each detected SE corresponded to one signal pulse $[5,6]$. We collected 32 image frames at a pixel resolution of 262 by 188 pixels, beam current of $2 \mathrm{pA}$, beam energy of $10 \mathrm{kV}$, and a pixel dwell time of $440 \mathrm{~ns}$. Each image frame had the SEM scan waveform and the in-lens and in-chamber SE detector signal with the same time axis. We wrote code to segment the detector signal into pixels, count the number of SEs in each pixel, and create a reference image using counts from both detectors and all frames. To implement CR our code looked at the number of SEs (hereafter referred to as NSE-limited CR) or the number of illuminations (hereafter referred to as $M$-limited CR) after every frame. For the $M$-limited scheme, if the number of illuminations crossed a maximum threshold, we did not use the counts from the following frames. For the NSE-limited scheme, if the number of SEs for a pixel crossed a maximum threshold, we did not use SE counts for that pixel in the following frames. Therefore, the incident electron dose per pixel was uniformly distributed over all pixels for the $M$-limited scheme and non-uniformly distributed for the NSE-limited scheme. To compare the CR images to the reference SE count image, our code scaled the SE counts for each pixel in the CR images by the ratio of the maximum number of frames (32) to the number of frames used at that pixel in the CR image. We used two metrics to quantify the comparison between CR images - Thong's signal-to-noise-ratio (SNR) [7] and mean absolute relative error (MARE) [8] which is the mean of the absolute value of the difference between the CR image and the reference image, expressed as a fraction of the mean of the reference image.

Figure 1(a) is a conventional SEM image of a 120 um-period copper mesh suspended in vacuum. This image has an SNR of 7.9. Figure 1(b) is an SE count reference image of the same sample. The color bar to the right indicates the correspondence between pixel grayscale level and number of SEs, and we scaled the conventional image (a) so that its mean intensity was the same as the mean counts in the SE count image (b). This image has an SNR of 11.2, 30\% higher than the conventional image. Figure 1(b) is an $M$ limited CR generated with threshold $M=14$. This image has an SNR of 5.73 and a MARE of 0.21. In this image the average incident electron dose on the sample is 56\% lower than that in figure 1(a). Figure 1(c) is an NSE-limited CR image generated with threshold $N_{\mathrm{SE}}=8$. This image has an SNR of 5.68 and a MARE of 0.18 . In this image the average dose is $57 \%$ lower than that in the reference image. We can see that the two schemes have comparable SNR, and the NSE-limited scheme has better MARE at the same dose. 
In figure 2 we plot SNR (2(a)) and MARE (2(b)) as a function of the fraction of the total incident electron dose on the sample for the reference image. We can see that both schemes have comparable SNR, and the

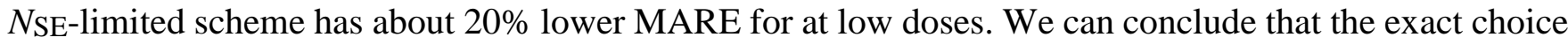
of CR scheme would depend on the type of sample and error metric important in the experiment. Future work will focus on using recent implementation of ns beam-blanking on the SEM [9] to implement online SE count imaging and CR [10].

a

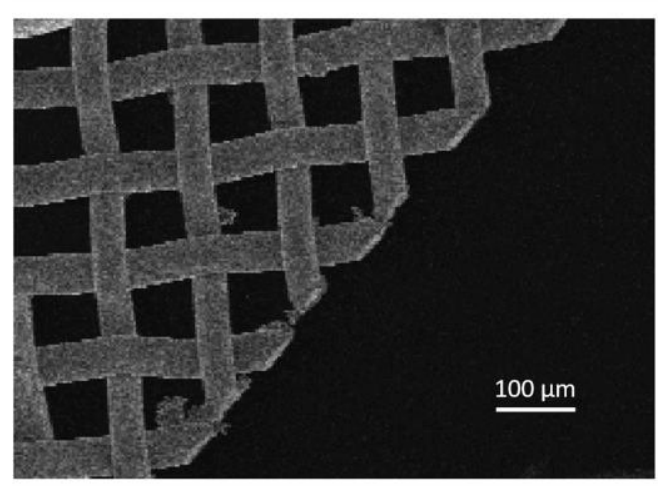

C

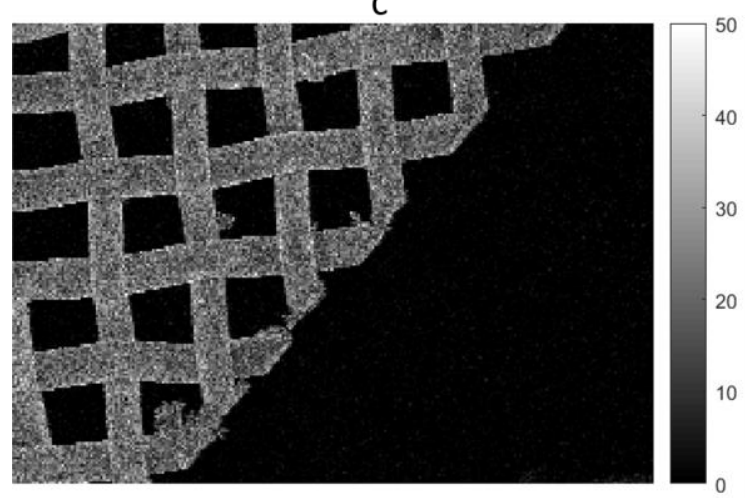

b

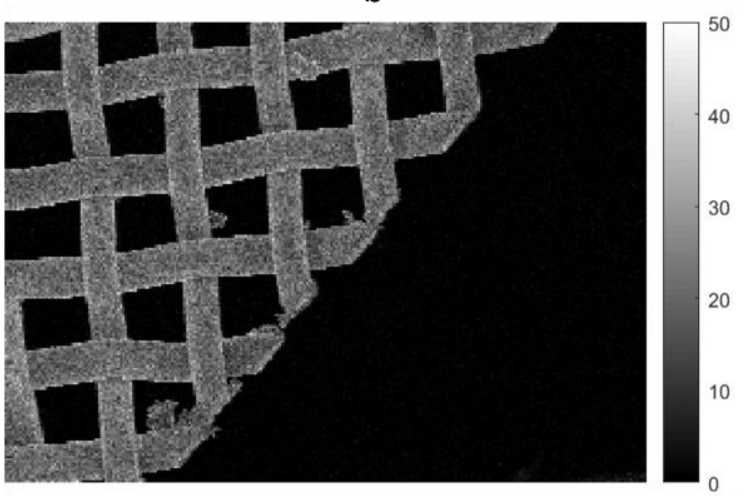

d

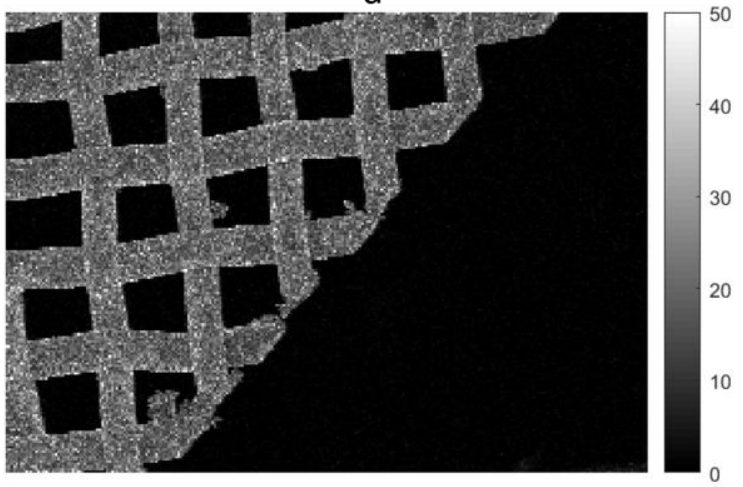

Figure 1. SE count imaging and CR in SEM. (a) Conventional SEM image of a $120 \mu \mathrm{m}$-period copper mesh suspended over vacuum, with an SNR of 7.9. (b) SE count image of the same sample at the same dose, with an SNR of 11.9. (c) M-limited CR image with SNR=5.73, MARE=0.21 and 56\% lower dose than (b). (d) NSE-limited CR image with $\mathrm{SNR}=5.68, \mathrm{MARE}=0.18$ and 57\% lower dose than (b).
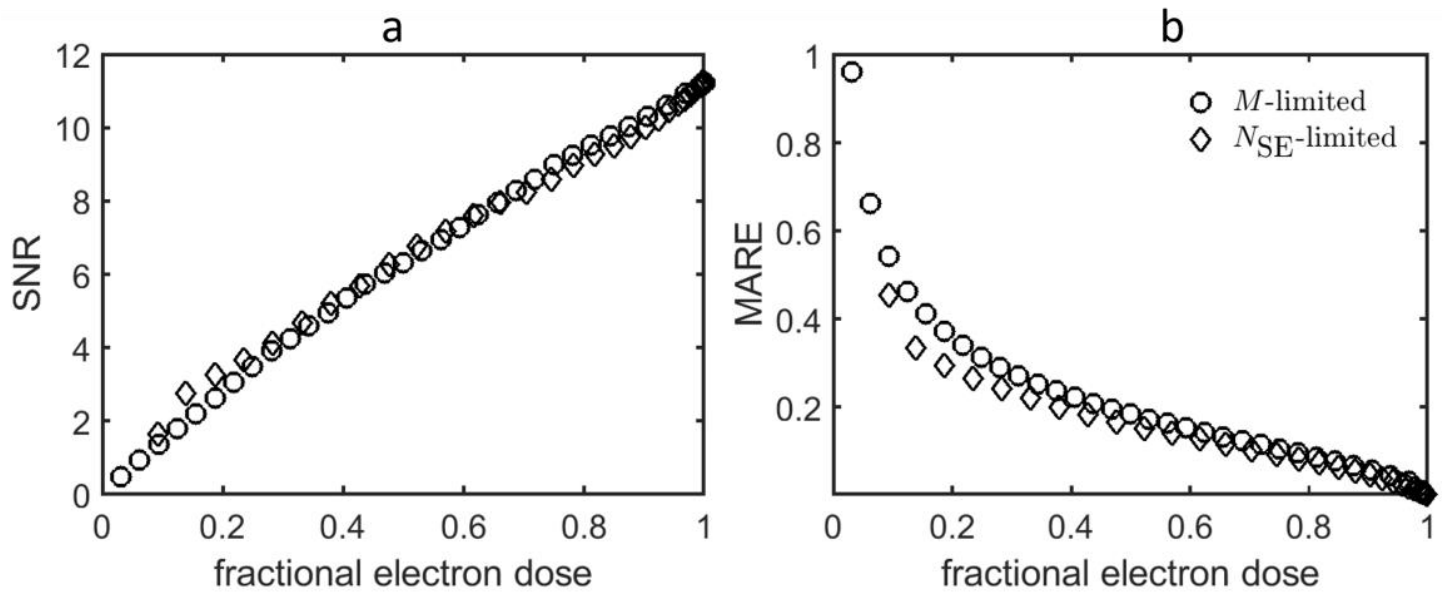
Figure 2. SNR (a) and MARE (b) for the two CR schemes as a function of the relative incident dose. NSElimited CR has comparable SNR and lower MARE than M-limited CR.

\section{References}

[1] Stevens, L. Luzi, H. Yang, L. Kovarik, B. L. Mehdi, A. Liyu, M. E. Gehm, and N. D. Browning, Appl. Phys. Lett. 112, 043104 (2018).

[2] T. Dahmen, M. Engstler, C. Pauly, P. Trampert, N. De Jonge, F. Mücklich, and P. Slusallek, Sci. Rep. 6, 25350 (2016).

[3] Y. Jiang, Z. Chen, Y. Han, P. Deb, H. Gao, S. Xie, P. Purohit, M. W. Tate, J. Park, S. M. Gruner, V. Elser, and D. A. Muller, Nature 559, 343 (2018).

[4] A. Agarwal, K. K. Berggren, Y. J. van Staaden, and V. K. Goyal, Phys. Rev. A 99, 063809 (2019).

[5] D. C. Joy, in Biol. Low-Voltage Scanning Electron Microsc. (Springer New York, New York, NY, 2007), pp. 129-144.

[6] Y. Uchikawa, K. Gouhara, S. Yamada, T. Ito, T. Kodama, and P. Sardeshmukh, J. Electron Microsc. (Tokyo). 41, 253 (1992).

[7] J. T. L. Thong, K. S. Sim, and J. C. H. Phang, Scanning 23, 328 (2001).

[8] C. Sammut and G. I. Webb, editors, in Encycl. Mach. Learn. (Springer US, Boston, MA, 2011), pp. 652-652.

[9] R. J. Moerland, I. G. C. Weppelman, M. W. H. Garming, P. Kruit, and J. P. Hoogenboom, Opt. Express 24, 24760 (2016).

[10] The authors acknowledge funding from the Gordon and Betty Moore Foundation, and the U.S. NSF under grands 1422034 and 1815896, and useful discussions with the QEM-2 collaboration. 